\title{
Morphology development and size control of poly(trimethylene terephthalate) nanofibers prepared from poly(trimethylene terephthalate)/cellulose acetate butyrate in situ fibrillar composites
}

\author{
M. F. Li • R. Xiao • G. Sun
}

Received: 20 October 2010/ Accepted: 28 January 2011 / Published online: 15 February 2011

(c) The Author(s) 2011. This article is published with open access at Springerlink.com

\begin{abstract}
Formation of nano-fibrillar composite structures provides an effective method for preparing thermoplastic nanofibers. By mixing two immiscible thermoplastic polymers in a twin screw extruder, poly(trimethylene terephthalate) (PTT) formed nano-fibrillar morphology in cellulose acetate butyrate (CAB) matrix, and then PTT nanofibers were obtained from PTT/CAB in situ fibrillar composites after removing the matrix phase of $\mathrm{CAB}$. Blend ratio, shear rate, and draw ratio were three important parameters in the extrusion process, which could affect the shape and size of nanofibers. By varying the process conditions, average diameter of PTT nanofibers could be controlled in the range of $80-400 \mathrm{~nm}$. Besides this, the mechanism of nano-fibrillar formation in PTT/CAB blends was also studied by collecting samples at different stages in the extruder. The morphology developmental trends of PTT dispersed phase with different blend ratios were nearly the same. From initial to metaphase and later phase development, the PTT dispersed component undergo the formation of sheets, holes, and network structures, then the size reduction and formation of nanofibers.
\end{abstract}

\section{F. Li · R. Xiao $(\bowtie) \cdot$ G. Sun}

State Key Laboratory for Modification of Chemical Fibers and Polymer Materials, College of Materials Science and Engineering, DongHua University, Shanghai 201620,

People's Republic of China

e-mail:xiaoru@dhu.edu.cn

G. Sun

Fiber and Polymer Science, University of California, Davis, Davis, CA 95616, USA

\section{Introduction}

Polymer blends have been increasingly used in a wide variety of applications since they can meet a vast range of performance demands and provide unique properties. Most properties of immiscible polymer blends depend on the phase morphology, such as spheres, sheet, and fibers. Spheric structures can increase the impact resistance of the matrix materials, whereas sheet-like inclusions have the ability to greatly enhance the barrier properties of a film and fibers, which can significantly increase unidirectional strength of the materials $[1,2]$. Furthermore, by having a correct combination of manufacturing and post-processing conditions, it is possible to generate particular morphology that can meet the peculiar needs [3].

Among the mentioned morphologies of the polymer blends, in situ fibrillar phase morphology has been extensively investigated. Based on certain conditions, one material can exist in the other material as the form of fibers, socalled in situ fibrillar blends. These materials, however, mainly focused on the in situ composites with improved mechanical properties, such as polypropylene/poly(ethylene terephthalate), thermotropic liquid crystal polymers/ poly(ethylene terephthalate), polycarbonate/high density polyethylene, polypropylene/polyamide-6, polypropylene/ polystyrene, and so on [4-10]. Besides this, in situ composites also provide a method to prepare nanofibers. Fakirov further developed the concept of microfibrillar composites (MFC), prepared a new type of polymer-polymer composite from polymer blends where the isotropic matrix is reinforced by nanofibrils (nanofibrillar composite NFC). They obtained the neat PET nanofibers and suggested the potential applications of this nanofibrillar material [11-14]. Moreover, isotactic polypropylene, high density polyethylene, poly(trimethylene terephthalate), and poly(ethylene-co-(glycidyl 
methacrylate)) nano-scale fibers were also successfully produced by using this method [15-18].

Poly(trimethylene terephthalate) (PTT) was chosen as a dispersed component in this study, which is one of the most important polymer materials for engineering plastic and textile fiber. Cellulose acetate butyrate $(\mathrm{CAB})$ was chosen as the matrix, as it is immiscible with many thermoplastic polymers and can be removed easily by acetone. During melt extrusion in a twin screw extruder, a series of deformation of dispersed polymer will happen, making PTT form nanofibrills in the $\mathrm{CAB}$ matrix. After removal of the matrix, PTT nanofibers can be obtained. The dispersed fibrillar phase can be affected by blend ratio, shear rate, and draw ratio. Therefore, the size of obtained nanofibers can be easily controlled by adjusting these parameters.

Many researchers have studied the morphology development of polymer blends and the effect of various parameters on it [19-27]. Macosko studied morphology development during the initial stages of polymer-polymer blending, and give the proposed mechanism for initial morphology development [28-30]. Favis investigated the effect of mixing time on the morphology development of blends of polypropylene and polycarbonate and concluded that the most significant changes in morphology occurred during the first 2 min of mixing when melting and softening of the materials was also occurring [31]. Jana studied the effects of viscosity ratio and composition on development of morphology in chaotic mixing of polymers $[32,33]$. Sundararaj studied the effect of compatibilization and rotation rate on the morphology development of polymer blends. Many of those researches showed that the final size and shape of the dispersed phase will not only depend on the processing conditions but also on the physical properties of each component [34].

In this article, PTT nanofibers were prepared by in situ fibrillar composites. In order to control the size of PTT nanofibers, several factors in the extrusion process were studied, such as blend ratio, shear rate, and draw ratio. It is the first time that the articles focus on the effects of processing parameters on the shape and size of nanofibers obtained by this method. Furthermore, for understanding the formation mechanism of PTT nanofibers, the morphology evolvement of the dispersed phase, form the initial particles to the final nanofibers, were also analyzed in this study.

\section{Experiment}

\section{Materials}

Cellulose acetate butyrate (CAB) (CAB-381-20, butyryl content $37 \mathrm{wt} \%$; acetyl content $13.5 \mathrm{wt} \%$; hydroxyl content
$1.8 \mathrm{wt} \%$ ) was purchased from Eastman Chemical Company. Its melting point is $195-205{ }^{\circ} \mathrm{C}$; Molecular Weight is 70000 . $\mathrm{CAB}$ was used as a matrix. Poly(trimethylene terephthalate) (PTT, CORTERRA), provided by the Shell Chemicals Europe B.V. PTT was used as a disperse component. Its melting point is $225-228{ }^{\circ} \mathrm{C}$; Density is $1.3-1.44 \mathrm{~g} / \mathrm{cm}^{3}$.

\section{Preparation}

All of the materials were dried in a vacuum prior to mixing and melting blending. The blends were prepared using a co-rotating twin screw extruder $(D=16 \mathrm{~mm}, L / D=40$, EUROLAB16, Thermo-Haake Co.). The extruder barrel had 10 heating zones, the barrel temperature profiles were $220,230,235,240,240,240,235,230,225$, and $240{ }^{\circ} \mathrm{C}$, respectively.

In the study of the influence of blend ratio on the average diameter of PTT nanofibers, PTT mixed with $\mathrm{CAB}$ at different blend ratio of 10/90, 20/80, 30/70, 40/60, and $50 / 50$, at a shear rate of $30 \mathrm{~s}^{-1}$ and a draw ratio of 25 . In the study of the influence of shear rate, PTT mixed with $\mathrm{CAB}$ at different shear rate of $10,30,50,80,100$, and $120 \mathrm{~s}^{-1}$, with the same blend ratios of 20/80 and draw ratio of 25. In the study of the effect of draw ratio, PTT mixed with $\mathrm{CAB}$ at the same blend ratio of $20 / 80$ and shear rate of $30 \mathrm{~s}^{-1}$, with different draw ratio (the area of cross-section of the die to that of the extrudates) of $1,2,5,15,25$, and 60 , respectively.

There are three different sampling points in the extruder. The melting behavior and morphology evolution of the blends along the extruder was studied through the examination of samples collected at different zones of the extruder. The samples, taken from the extruder, were immediately cooled in iced water to preserve the morphology that developed in the extruder. The matrix component was removed by immersing the samples in acetone at room temperature with the acetone exchanged every 30 min till the matrix was removed completely.

\section{Characterization}

The complex viscosity, elastic modulus of the polymers used in this study were determined by using a dynamical rheometer (ARES-RFS) with a $25 \mathrm{~mm}$ parallel plate. The rheological measurements of PTT and CAB were performed at $240{ }^{\circ} \mathrm{C}$.

The PTT/CAB blends taken from three different zones of the extruder were fractured in liquid nitrogen. The fracture surfaces of the blends were observed using a scanning electron microscope (SEM).

After removing the matrix component, the PTT dispersed component samples were observed using SEM. The 
diameter distributions and averages were obtained by measuring 100 nanofibers. The number averaged diameters were calculated as follows (Eq. 1).

$D_{\mathrm{N}}=\frac{\sum N_{\mathrm{i}} D_{\mathrm{i}}}{\sum N_{\mathrm{i}}}$

where $D_{\mathrm{N}}$ is the number averaged diameter and $N_{\mathrm{i}}$ is the number of nanofibers with a diameter of $D_{\mathrm{i}}$.

\section{Results and discussion}

Formation mechanism

The formation of PTT nanosized fibers in the twin-screw extruder was an overall result of continuous deformation, elongation, orientation and coalescence of dispersed micelles to nanofibers in CAB matrix. Viscosity ratio $\left(\eta_{\mathrm{d}} / \eta_{\mathrm{m}}\right.$, $\eta_{\mathrm{d}}$ is the viscosity of the dispersed component, $\eta_{\mathrm{m}}$ is the viscosity of the matrix component) played an important role on the deformation of the dispersed phase. The effect of viscosity ratio $\left(\eta_{\mathrm{d}} / \eta_{\mathrm{m}}\right)$ on the fibril formation has been analyzed largely. Fibrils were reported at $0.3<\eta_{\mathrm{d}} / \eta_{\mathrm{m}}<1.0$ for polyethylene/polystyrene blends, at $\eta_{\mathrm{d}} / \eta_{\mathrm{m}}>3.7$ for poly (ethylene terephthalate)/polyamide blends and at $\eta_{\mathrm{d}} / \eta_{\mathrm{m}}<1$ for polypropylene/ethylene-propylene copolymer blends [35]. Plate et al. studied 13 different pairs of polymers and indicated that good fibrillation can be achieved when the viscosity ratio in the range of $0.1<\eta_{\mathrm{d}} / \eta_{\mathrm{m}}<10[36,37]$.

For studying the rheological properties of all the materials used in this study, the complex viscosity $\left(\eta^{*}\right)$ and elastic modulus $\left(G^{\prime}\right)$ of PTT and CAB as a function of frequency at $240{ }^{\circ} \mathrm{C}$ are shown in Fig. 1a. Figure $1 \mathrm{~b}$ gives the corresponding viscosity ratio and elasticity ratio $\left(G_{\mathrm{d}}^{\prime} / G_{\mathrm{m}}^{\prime}, G_{\mathrm{d}}^{\prime}\right.$ is the elastic modulus of the dispersed component, $G_{\mathrm{m}}^{\prime}$ is the elastic modulus of the matrix component) of the PTT/CAB system. With the increasing of the shear rate, the complex viscosity decreased continuously, implying that the PTT and CAB were non-Newtonian fluids and all followed the shear thinning behavior. For the system of PTT/CAB, the viscosity ratio was from 1.6 to 2.2. Therefore, fibrillar dispersed phase can be obtained after removal of the matrix component. Beside this, the elasticity ratio was changed from 0.2 to 0.9 , the stability of the dispersed fibers was enhanced by the elasticity of materials.

When the mixed materials were feed into the twin screw extruder, a serious of deformation will happen [38], making the dispersed phase transformed from pellets to the final nano-sized fibers. Figure 2 shows the morphology development of the PTT dispersed phase with different blend ratios after the polymer blends were taken from different sampling points in the extruder and removal of the
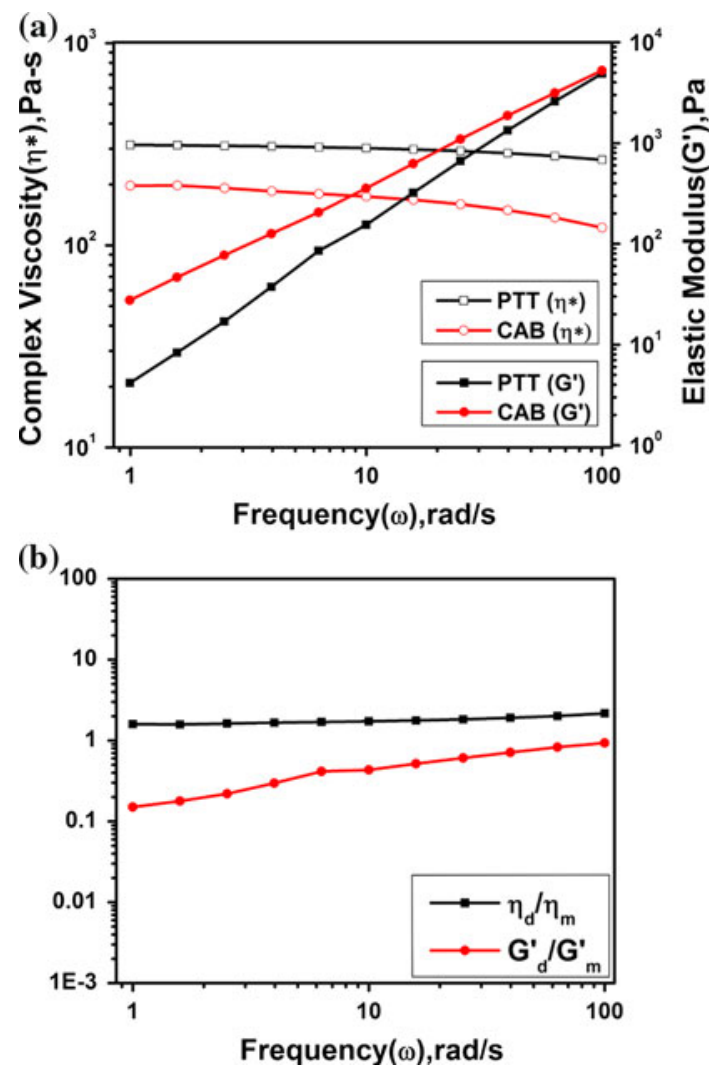

Fig. 1 Frequency dependence of a viscosity and elastic modulus for PTT and $\mathrm{CAB}$ at $240{ }^{\circ} \mathrm{C}, \mathbf{b}$ viscosity ratio and elasticity ratio of PTT/ CAB system

matrix. As shown in Fig. 2, two different representative SEM images can be observed from each sampling location. Samples $(1,2),(3,4)$, and $(5,6)$ displayed the morphology of initial, metaphase and later phase development of PTT dispersed phase, respectively.

The initial morphological development of PTT with different blend ratios showed remarkably similar types of structures. The quick morphological changes in the initial stage of blending are caused by the "sheeting" mechanism $[26,28]$. This mechanism involves the formation of sheets or ribbons when a large piece of dispersed component was dragged across the hot surface. As a result of the effect of shear flow and interfacial tension, the sheets were unstable and began to break up resulting in formation of holes in them, the holes were filled with the matrix component. When the holes attained sufficient size and concentration, they would coalesce and form network structures, as shown in sample $(1,2)$ of Fig. 2. Therefore, the initial morphology development procedure for the dispersed phase of PTT in the matrix was from pellets to sheets or ribbons, then formed holes and network structures. Sample $(3,4)$ in Fig. 2 show the metaphase development of PTT dispersed phase. It can be seen that massive fibers formed by breaking up of the network structures, as an enormous 


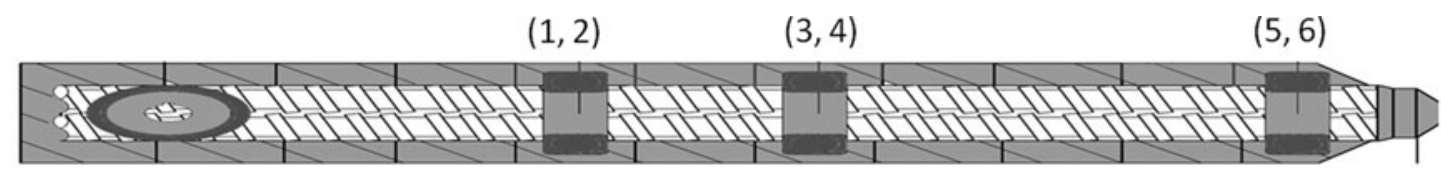

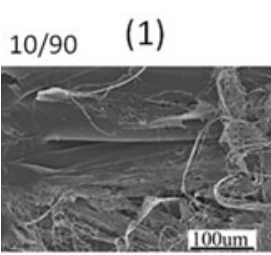

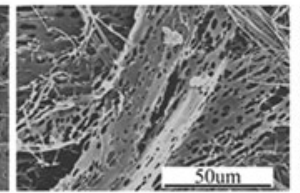

$20 / 80$

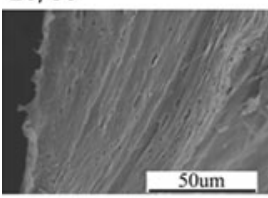

$30 / 70$

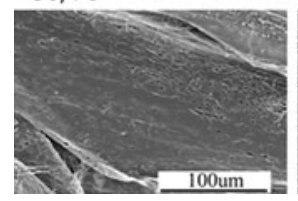

(2)

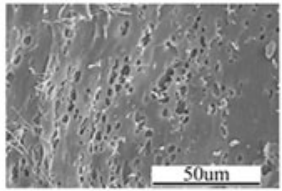

(3)
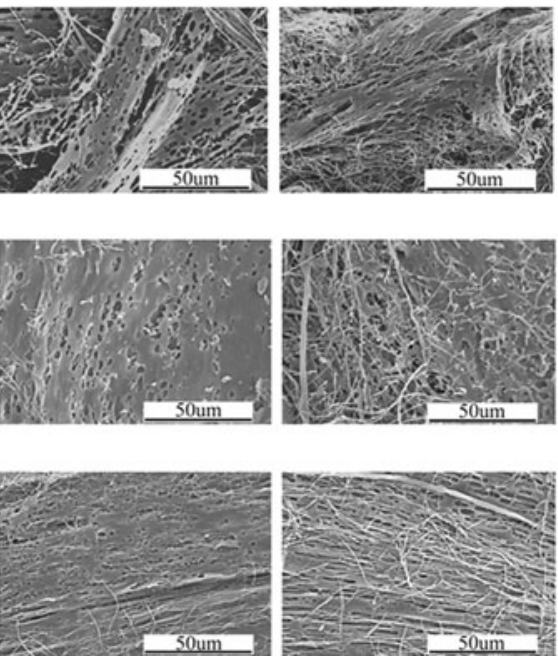

(4)
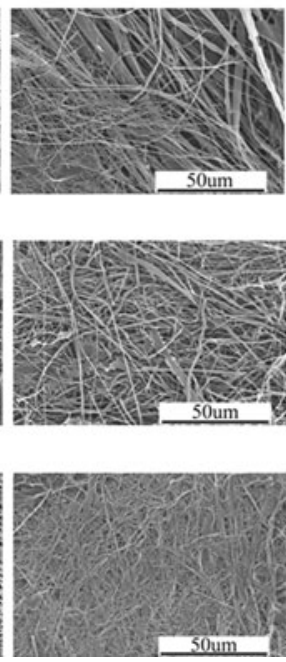

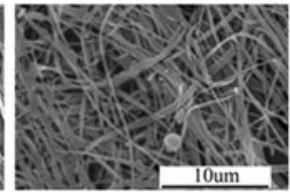

(5)
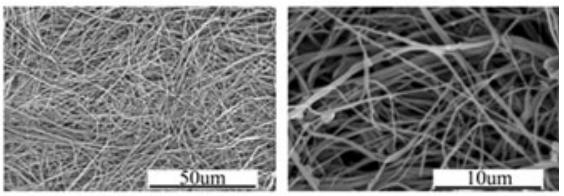

(6)
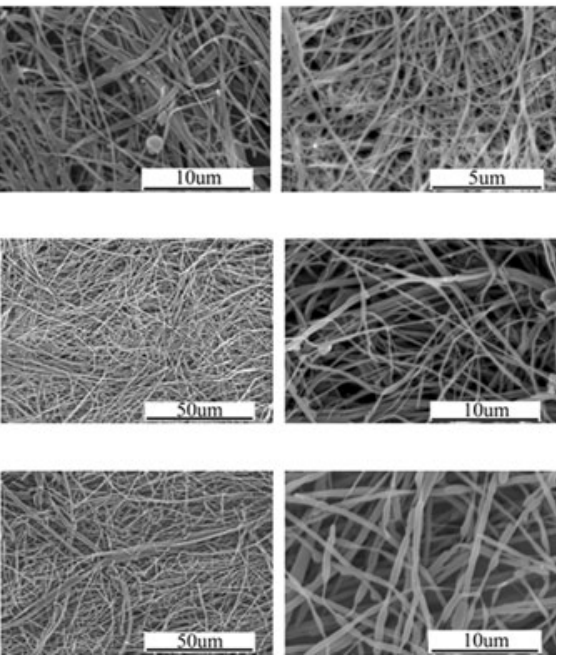

Fig. 2 Morphology development of PTT dispersed phase in the twin extruder

reduction in phase size took place from sample $(1,2)$ to sample $(3,4)$. Therefore, the metaphase development of the polyester dispersed phase can be described as the formation of fibers. Sample $(5,6)$ in Fig. 2 displays the later morphological development of the PTT dispersed phase. It can be seen that nanofibers were further developed in the later stage.

\section{Effect of blend ratio}

After extruded from the twin screw extruder, PTT/CAB composite fibers can be obtained. Blend ratio of PTT/CAB has been found an important factor in controlling the phase shapes and size of the polymer blends during deformation processing [25]. Figure 3 gives the fracture surface of PTT/ $\mathrm{CAB}$ composite fibers with different blend ratios. It is clear that the blends of PTT/CAB with the blend ratios of $10 / 90$, 20/80, and 30/70 showed a typical droplet-in-matrix morphology. When the concentration of PTT increased to $40 \mathrm{wt} \%$, the PTT droplet began to collided with each other, leading to the formation of a small quantity of continuous plate structure. With the increase of the percentage of PTT dispersed component, the probability of particle collision increased synchronously. At the blend ratio of PTT/CAB $50 / 50$, a type of co-continuous structures can be observed from the fracture surface of PTT/CAB blends, shown in Fig. 3.

PTT dispersed phase can represent different morphologies after removal of the $\mathrm{CAB}$ matrix from the $\mathrm{PTT} / \mathrm{CAB}$ composite fibers in different blend ratios. As shown in Fig. 4, at the blend ratios of PTT/CAB 10/90, 20/80, and 30/70, PTT nanofibers could be obtained after removal of the $\mathrm{CAB}$ matrix, respectively. At the blend ratio of PTT/ CAB 40/60, fibers could also be obtained, however, a spot of plate existed between the fibers as the coalescence of PTT component. Even some CAB matrix, which was wrapped by PTT, could not be removed completely. When the concentration of PTT increase to $50 \mathrm{wt} \%$, PTT fibers could not be obtained, due to the fact that both polymers formed co-continuous structures in the composite fibers.

Figure 5 shows the average diameter evolution of PTT nanofibers as a function of blend ratios. With the increase of PTT percentage from 10 to $40 \mathrm{wt} \%$, the diameter of PTT nanofibers were 98, 110, 151, and $268 \mathrm{~nm}$, respectively. As expected, the PTT nanofibers showed an increase in the average diameter, along with the broadening of the diameter distribution, as the percentage of PTT dispersed component increases. This is mainly caused by the PTT droplet coalescence during melt-extruding, which is known to be a random process and enhanced by the increase of the blend ratio, hence broadening the PTT nanofiber size distribution.

\section{Effect of shear rate}

Shear rate is an important parameter of the formation of nanofibrills in the twin screw extruder. It acts on the deformation of the dispersed phase and can affect the 

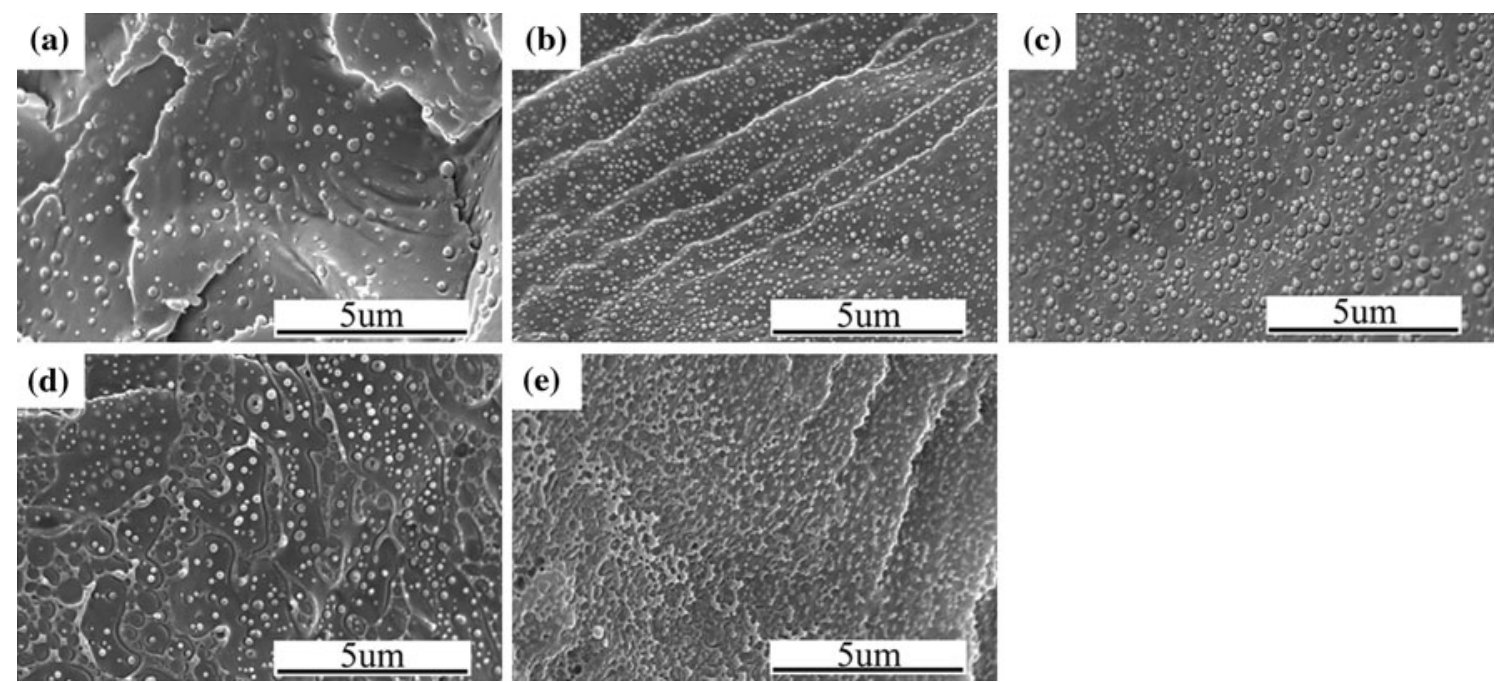

Fig. 3 Fracture surface of PTT/CAB composite fibers with different blend ratios a 10/90, b 20/80, c 30/70, d 40/60, e 50/50
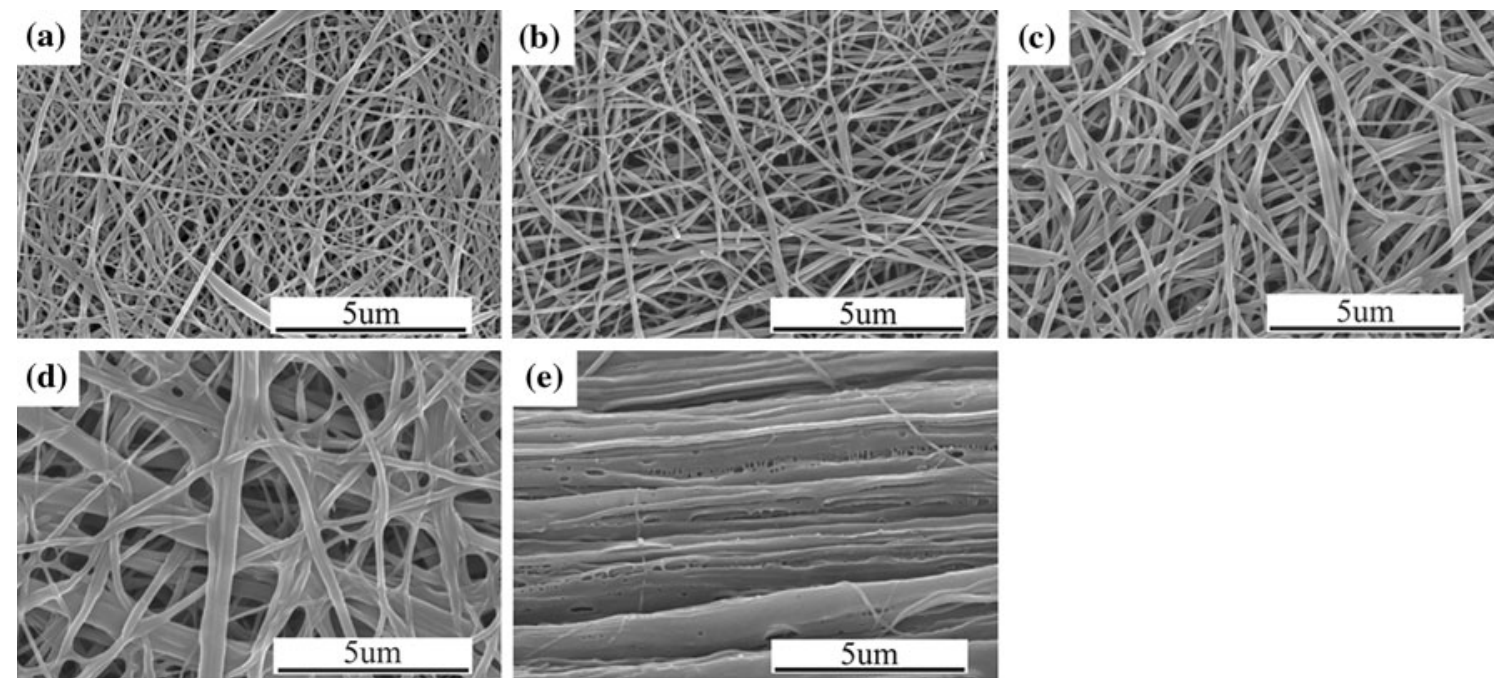

Fig. 4 Morphology of PTT dispersed phase obtained from PTT/CAB polymer blends with different blend ratios a 10/90, b 20/80, c 30/70, d 40/ 60 , e $50 / 50$

viscoelasticity of the polymer blends during the melting process [39]. It is well known that higher shear stress is helpful for the deformation of the droplet, while increasing the screw speed can also increase the probability of coalescence, leading to the increasing in dispersed particle size. Therefore, the effect of the shear rate on the shape and size of dispersed phase is multiple. Figure 6 gives the morphology of nanofibers obtained from PTT/CAB blends with different shear rates, and Fig. 7 shows the corresponding size of PTT nanofibers. The average diameter of nanofiber with the shear rate of $10,30,50,80,100$, and $120 \mathrm{~s}^{-1}$ were $103,110,104,109,106$, and $104 \mathrm{~nm}$, respectively. Clearly, shear rate did not affect the size of formed nanofibers, and the average diameter of PTT nanofibers nearly stable with the increase of shear rate.
This is maybe because the multiple influence of shear rate on the dispersed phase, the deformation and coalescence of droplet were balanced out during the extrusion process. With the increase of shear rate, the viscosity ratio of PTT/ $\mathrm{CAB}$ system did not change too much. Therefore, the diameter of PTT nanofibers were not affected so much by the shear rate.

\section{Effect of draw ratio}

It is well known that an elongation flow is beneficial to the fibrillation of the dispersed phase particles in an immiscible blend, so draw ratio is also an important factor in controlling the phase shapes and size of the polymer blends during the deformation processing [40,41]. Figure 8 shows 
Fig. 5 Effect of blend ratio on the average diameter of PTT nanofibers, with blend ratio of a $10 / 90$, b $20 / 80$, c $30 / 70, d$ $40 / 60$
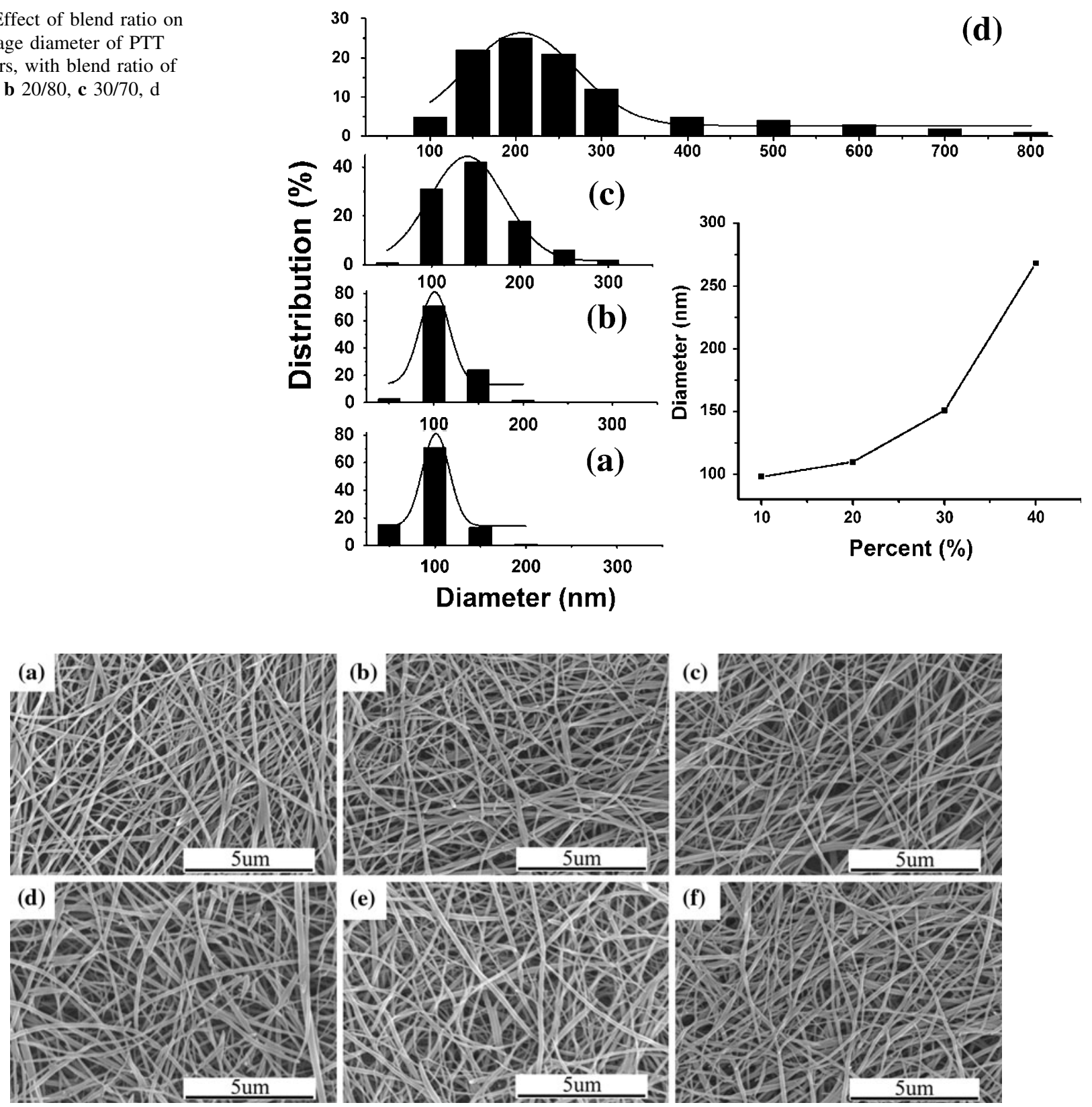

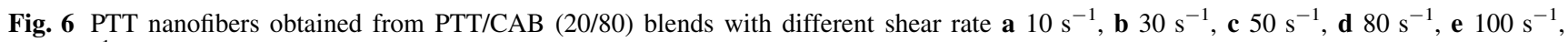
f $120 \mathrm{~s}^{-1}$

the fracture surface of PTT/CAB composite fibers with different draw ratio. Figure 9 gives the average diameter of the nanofibers obtained after removing the matrix component of CAB. Obviously, the size of dispersed sphere with higher draw ratio is much smaller. The average diameter of the obtained nanofibers with the draw ratios of 1,2, 5, 15, 25 , 60 were $325,320,175,125,110$, and $80 \mathrm{~nm}$, respectively. Higher draw ratio provide higher elongation flow to the polymer blends, it is conducive to the stretching and orienting of the microfibrils among the matrix component. Therefore, with the increase of the draw ratio, the average diameters of the obtained nanofibers reduce simultaneity.
However, the size of PTT nanofibers tended to stable when the draw ratio beyond a certain value. As shown in Fig. 9, less than draw ratio of 15 , the diameter of nanofibers decreased remarkably. After the draw ratio lager than 15, the diameter still decreased though not so significantly.

\section{Conclusion}

Well-defined nanofibers were prepared from PTT/CAB immiscible blends, the effect of blend ratio, shear rate and draw ratio on the shape and size of PTT nanofibers were 
Fig. 7 Effect of shear rate on the average diameter of PTT nanofibers, with shear rate of a $10 \mathrm{~s}^{-1}$, b $30 \mathrm{~s}^{-1}$, c $50 \mathrm{~s}^{-1}$, d $80 \mathrm{~s}^{-1}$, e $100 \mathrm{~s}^{-1}$, f $120 \mathrm{~s}^{-1}$

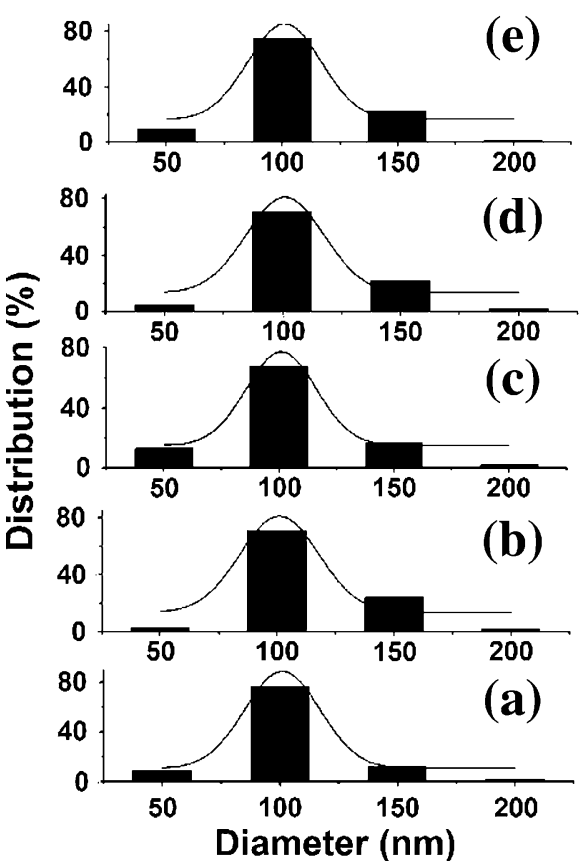

(f)

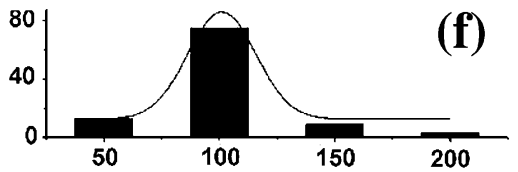

Diameter (nm)

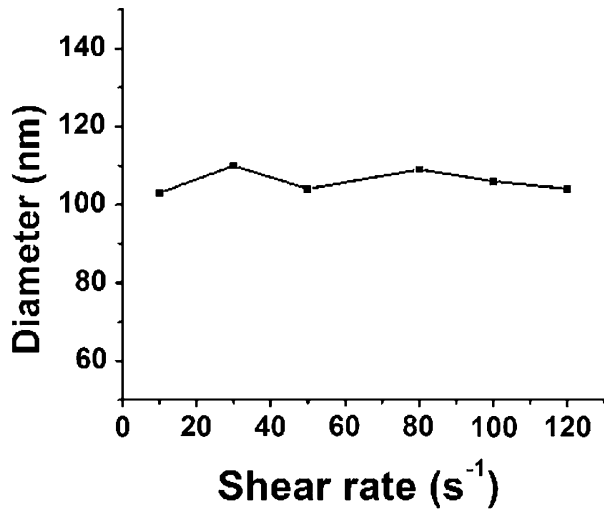

analysis in this study. PTT nanofibers can be obtained at the blend ratio of PTT/CAB 10/90, 20/80, and 30/70. At the blend ratio of PTT/CAB 40/60, the dispersed component began to coalescence, but fibers can still be obtained after removal of the $C A B$ matrix. When the blend ratio was increased to PTT/CAB 50/50, co-continuous structures were found. For the effect of draw ratio, less than draw ratio value of 15 , the diameter of nanofibers decrease remarkably, after the draw ratio lager than 15 , this decrease became less significant. The increase of shear rate was found to have no obvious effect on the final diameter of PTT nanofibers. Therefore, compared with shear rate, blend ratio, and draw ratio were more efficient at controlling the shape and size of PTT nanofibers.

Besides, for understand the formation mechanism of PTT nanofibers, morphology development of PTT dispersed phase were studied. The initial morphology development process for the dispersed phase of PTT was from pellets to sheets or ribbons, and then forms holes and network structures, follows the "sheeting" mechanism. The metaphase development of the PTT dispersed phase could be described as the formation of fibers. The later phase development of the PTT dispersed phase mainly involved the reduction in diameter of the fibers to the nano-scale.
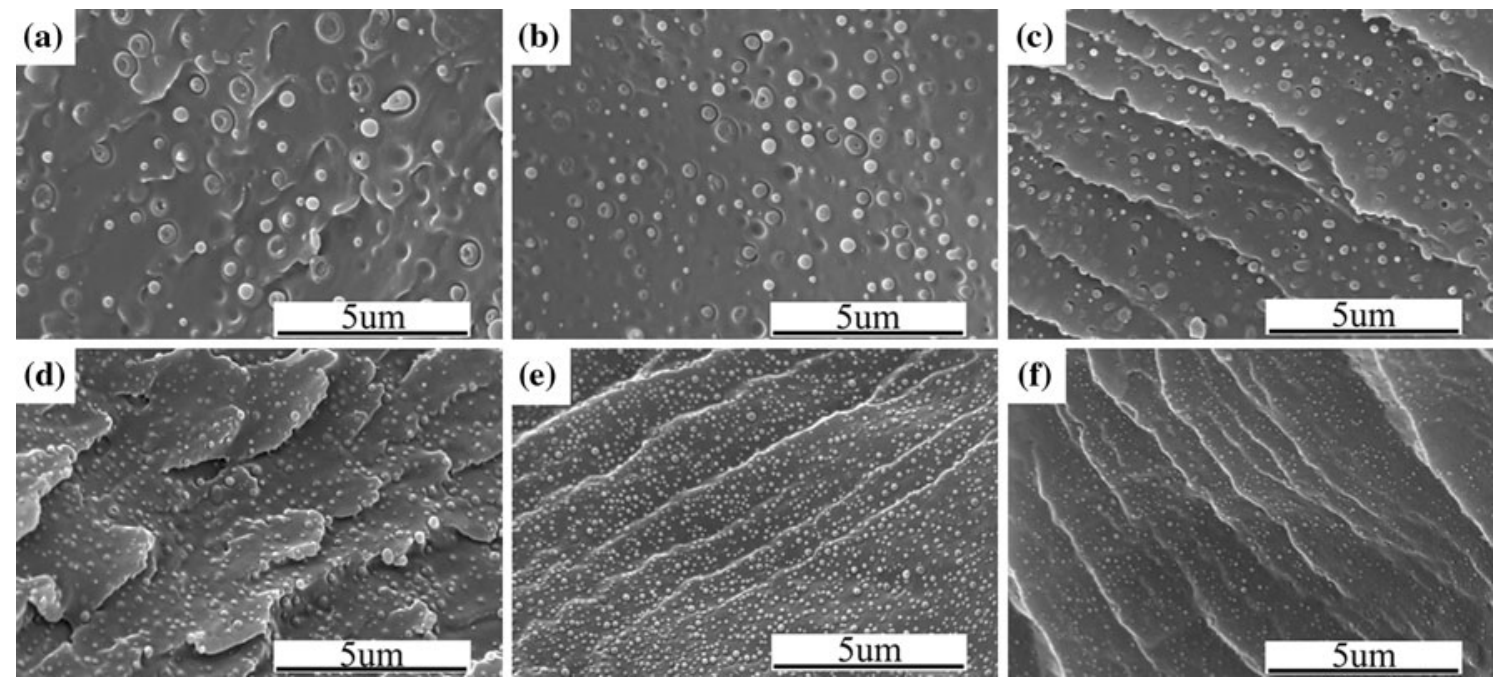

Fig. 8 Fracture surface of PTT/CAB (20/80) composite fibers with different draw ratio a 1, b 2, c 5, d 15, e 25, f 60 
Fig. 9 Effect of draw ratio on the average diameter of PTT nanofibers, with draw ratio of a 1, b 2, c 5, d 15 , e 25, d 60

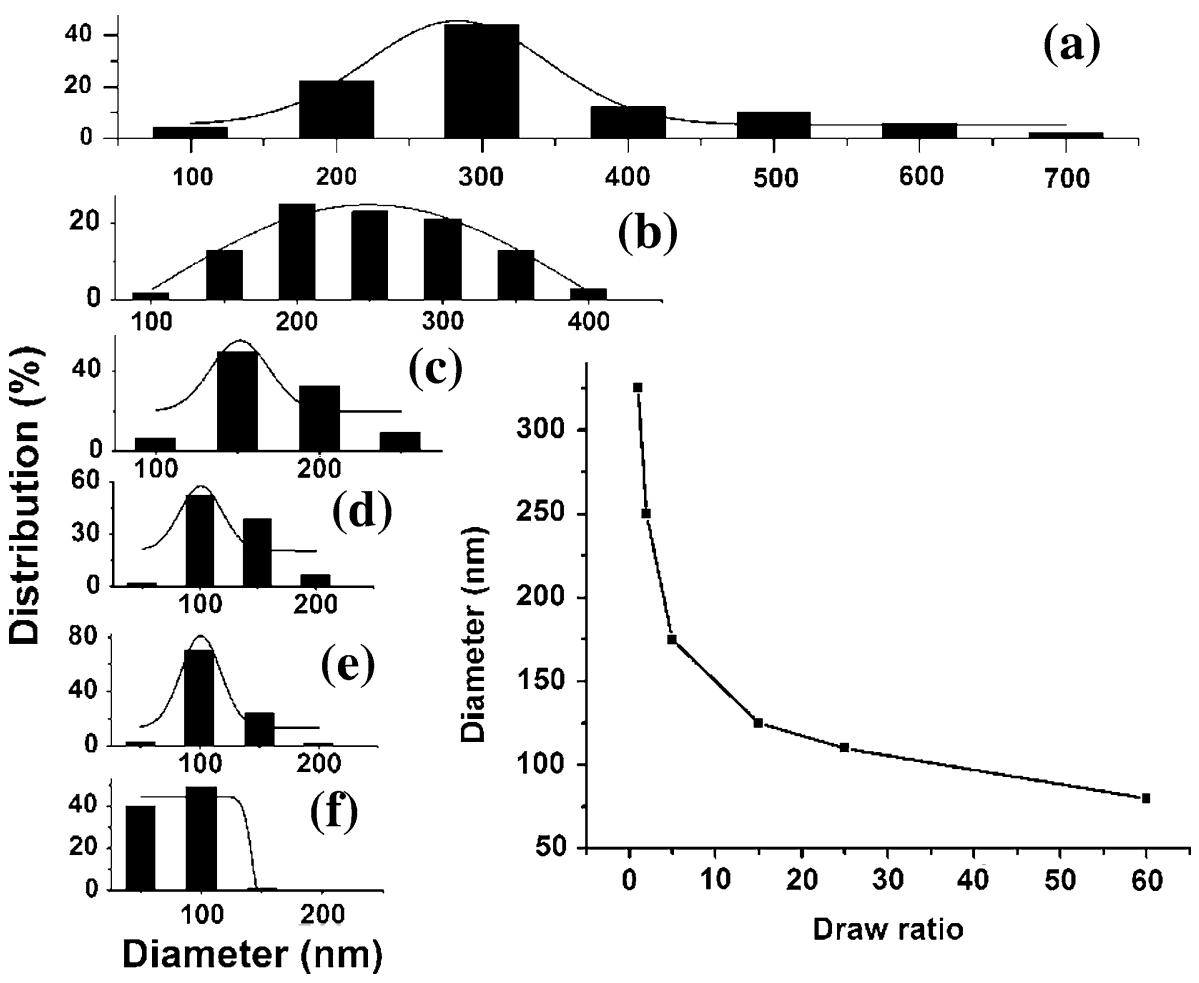

Acknowledgements This research was financially supported by the National Natural Science Foundation of China (No. 20874010), the program of Introducing Talents of Discipline to Universities (No. 111-2-04, B07024) and Assets Investment Company of Kunshan Development District, Jiangsu Province.

Open Access This article is distributed under the terms of the Creative Commons Attribution Noncommercial License which permits any noncommercial use, distribution, and reproduction in any medium, provided the original author(s) and source are credited.

\section{References}

1. Shields RJ, Bhattacharyya D, Fakirov S (2008) J Mater Sci 43:6758. doi:10.1007/s10853-008-2693-Z

2. Migler KB (2001) Phys Rev Lett $86: 1023$

3. Macosko CW (2000) Macromol Symp 149:171

4. Saikrasun S, Saengsuwan S (2009) J Mater Proc Technol 209:3490

5. Xu HS, Li ZM, Wang SJ, Yang MB (2007) J Polym Sci B 45:1205

6. Xu HS, Li ZM, Yang SY, Pan JL, Yang W, Yang MB (2005) Polym Eng Sci 45:1231

7. Xing Q, Zhu MF, Wang YH, Chen YM, Zhang Y, Pionteck J, Adler HJ (2005) Polymer 46:5406

8. Quan H, Li ZM, Zhong GJ (2007) J Macromol Sci B 46:285

9. Jayanarayanan K, Thomas S, Joseph K (2008) Composites A 39:164

10. Kim JY, Kim SH (2005) J Polym Sci B 43:3600

11. Fakirov S, Bhattacharyya D, Lin RJT, Fuchs C, Friedrich K (2007) J Macromol Sci 46:183

12. Duhovic M, Bhattacharyya D, Fakirov S (2010) Macromol Mater Eng 295:95

13. Fakirov S, Duhovic M, Maitrot P, Bhattacharyya D (2010) Macromol Mater Eng 295:515
14. Fakirov S, Bhattacharyya D, Shields RJ (2008) Colloids Surfaces A $313-314: 2$

15. Wang D, Sun G (2007) Eur Polym J 43:3587

16. Wang D, Sun G, Chiou BS (2007) Macromol Mater Eng 292:407

17. Wang D, Sun G, Chiou BS (2008) Macromol Mater Eng 293:657

18. Wang D, Sun G, Chiou BS, Hinestroza JP (2007) Polym Eng Sci 47:1865

19. Leblanc J, Pla F, Mercier M, Pitiot P (2009) Chem Eng Sci 64:1918

20. Huang WY, Shen JW, Chen XM, Chen HY (2003) Polym Int 52:1131

21. Schlatter G, Serra C, Bouquey M, Muller R, Terrisse J (2002) Polym Eng Sci 42:1965

22. Nair SV, Oommen Z, Thomas S (2002) J Appl Polym Sci 86:3537

23. Deyrail Y, Fulchiron R, Cassagnau P (2002) Polymer 43:3311

24. Li HX, Hu GH (2001) J Polym Sci B 39:601

25. Willemse RC, Ramaker EJJ, van Dam J, de Boer AP (1999) Polymer 40:6651

26. Lee JK, Han CD (1999) Polymer 40:6277

27. Everaert V, Aerts L, Groeninckx G (1999) Polymer 40:6627

28. Scott CE, Macosko CW (1995) Polymer 36:461

29. Sundararaj U, Dori Y, Macosko CW (1995) Polymer 36:1957

30. Scott CE, Macosko CW (1991) Polym Bull 26:341

31. Favis BD (1990) J Appl Polym Sci 39:285

32. Jana SC, Sau M (2004) Polymer 45:1665

33. Sau M, Jana SC (2004) Polym Eng Sci 44:407

34. Li HP, Sundararaj U (2009) Macromol Chem Phys 210:852

35. Kim BK, Do IH (1996) J Appl Polym Sci 60:2207

36. He JS, Bu WS, Zhang HZ (1995) Polym Eng Sci 35:1695

37. Platé NA, Kulichikhin VG, Talroze RV (1991) Pure Appl Chem 63:925

38. Lin B, Sundararaj U, Mighri F, Huneault MA (2003) Polym Eng Sci 43:891

39. Ghodgaonkar PG, Sundararaj U (1996) Polym Eng Sci 36:1656

40. Xu HS, Li ZM, Pan JL, Yang MB, Huang R (2004) Macromol Mater Eng 289:1087

41. Xue C, Wang D, Xiang B, Chiou BS, Sun G (2010) Mater Chem Phys 124:48 